\section{Mortalidade infantil por causas evitáveis no Brasil: um estudo ecológico no período 2000-2002}

\author{
Infant mortality from preventable causes in Brazil: \\ an ecological study in 2000-2002
}

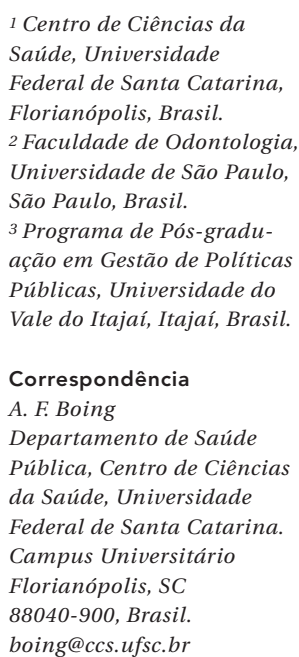

\begin{abstract}
This study aims to test the associations between infant mortality from preventable causes in Brazil and socioeconomic factors, including those pertaining to health services and investments. This was an ecological study using 296 Brazilian counties (municipalities) with more than 80,000 inhabitants each as the analytical units. Kruskall-Wallis and ANOVA tests were performed to compare independent variables according to infant mortality quartile, and Pearson and Spearman's correlation coefficients were computed to test the associations. As the infant mortality quartile from preventable causes increases, there is a gradual decrease in the municipal human development index, per capita gross domestic product, households with bathrooms and indoor plumbing, total health expenditures per inhabitant, and physicians per 1,000 inhabitants, and an increase in the Gini coefficient. Improved socioeconomic conditions and public health investments are strongly associated with reduction of infant mortality from preventable causes. This knowledge should permeate actions aimed at minimizing the number and unequal distribution of such deaths.
\end{abstract}

Infant Mortality; Infant Mortality Rate; Health Services; Socioeconomic Factors
Antonio Fernando Boing 1,2

Alexandra Crispim Boing 3

\section{Introdução}

Ao longo das últimas décadas a redução dos óbitos entre os menores de um ano de idade configurou-se como uma das principais metas na área da saúde em diversos países. Como conseqüência, uma acentuada redução na mortalidade infantil pôde ser observada. Esse declínio, ao serem analisados os dados de países dos cinco continentes, entre 1950 e 1994, chegou a $70 \%{ }^{1}$. Tendência de queda também foi observada no Brasil. Enquanto em 1980 morriam no país, em média, 82,8 crianças a cada mil nascidas vivas, em 2004 a taxa equivaleu a 26,6 2. A redução da taxa de fecundidade, a melhoria das condições gerais de vida e na provisão de serviços de saúde, além de políticas voltadas à saúde infantil são apontados como determinantes dessa tendência 3,4,5.

Ainda assim, o coeficiente de mortalidade infantil no Brasil permanece alto e o seu declínio em muitos momentos e lugares mascarou uma dilatação na diferença de óbitos entre pobres, com maiores taxas, e ricos 2,6. Tal desproporcionalidade é expressa no estudo de Wagstaff 7 que, ao analisar os dados de óbitos em menores de um ano de nove países em desenvolvimento, apontou o Brasil como detentor da maior desigualdade social na mortalidade infantil.

Muitas causas de óbitos infantis são consideradas evitáveis, ou seja, os conhecimentos e as tecnologias já existentes permitem intervenções eficazes de modo que tais condições jamais ou 
raramente evoluam a óbito. O estudo da distribuição do coeficiente de mortalidade infantil por causas evitáveis segundo condições sócio-econômicas e de serviços de saúde, pela natureza do próprio indicador, permite identificar injustiças sociais e fornece importantes subsídios para a implementação de políticas públicas equânimes e adequadas às distintas realidades do país. Diferenças nos valores do indicador entre grupos populacionais e regiões podem indicar que conhecidas medidas de prevenção de óbitos infantis não estão sendo empregadas de modo igualitário. Portanto, o objetivo desse estudo é investigar a associação entre indicadores sócio-econômicos e de serviços e investimentos em saúde e a mortalidade infantil por causas evitáveis nos municípios brasileiros com mais de 80 mil habitantes.

\section{Métodos}

Trata-se de um estudo ecológico, cujas unidades de análise foram os 296 municípios brasileiros que apresentaram no censo do ano 2000 população superior a 80 mil habitantes. A exclusão de municípios de menor porte procurou limitar a imprecisão nos coeficientes calculados, uma vez que o Sistema de Informações sobre Mortalidade (SIM) apresenta melhor qualidade de dados nas regiões mais desenvolvidas do Brasil 8, no contexto do país as capitais e cidades de médio porte ${ }^{9}$. Além disso, a taxa de mortalidade infantil por causas evitáveis apresenta grande oscilação em pequenas populações. Nesses casos, uma variação pequena no número absoluto de óbitos pode provocar grandes alterações na taxa de mortalidade. No presente estudo, foram selecionados os seguintes indicadores:

\section{Variável dependente}

\section{- Coeficiente de mortalidade infantil (CMI) por causas evitáveis}

Utilizou-se a classificação proposta pela fundação Sistema Estadual de Análise de Dados (Fundação SEADE) 10, que categoriza os óbitos infantis por causas evitáveis em: redutíveis por imunoprevenção; por adequado controle na gravidez; por adequada atenção ao parto; por ações de prevenção, diagnóstico e tratamento precoce e por intermédio de parcerias com outros setores. Para conferir maior estabilidade à variável, optou-se por empregar a média dela para os anos 2000, 2001 e 2002. Os óbitos foram obtidos junto ao SIM (http://tabnet.datasus.gov.br/cgi/ deftohtm.exe?sim/cnv/obtbr.def, acessado em 14/Mar/2006), e o número de nascidos vivos no Sistema de Informações sobre Nascidos Vivos (SINASC - http://tabnet.datasus.gov.br/cgi/ deftohtm.exe?sinasc/cnv/nvbr.def, acessado em 14/Mar/2006).

\section{Variáveis independentes}

\section{- Índice de Desenvolvimento Humano Municipal (IDHM)}

O índice abrange três dimensões: a educação, a longevidade e a renda dos municípios. Ele varia de 0 (nenhum desenvolvimento humano) a 1 (desenvolvimento humano total). Foi utilizado o índice referente ao ano 2000 extraído do Atlas do Desenvolvimento Humano no Brasil 11.

\section{- Coeficiente de Gini (Gini)}

Esse indicador expressa as desigualdades na distribuição de renda. Quanto maior o coeficiente, que varia de 0 a 1 , maior será a concentração de renda do município. O dado é do ano 2000 e oriundo do Atlas do Desenvolvimento Humano no Brasil 11 .

\section{- Produto Interno Bruto (PIB) per capita}

Representa a razão entre a totalidade da produção econômica em valores financeiros do município e a população global do mesmo. Foi utilizada a informação referente ao ano 200011.

\section{- Acesso a serviços básicos}

Como síntese dessa condição empregou-se a proporção de pessoas que viviam em domicílios com banheiro e água encanada em 200011.

\section{- Estabelecimentos de saúde por $10 \mathrm{mil}$ habitantes}

Conforme metodologia da Pesquisa de Assistência Médico-Sanitária, foram considerados como estabelecimentos de saúde todos os locais que possuíam um mínimo de técnica apropriada para o atendimento rotineiro à população, como postos de saúde, hospitais, prontos-socorros etc. Os valores são referentes ao ano 2002 e utilizados como proxy da estrutura de assistência à saúde instalada no município (http:/ / www.ibge.gov.br/ home/estatistica/populacao/condicaodevida/ ams/default.shtm, acessado em 20/Mar/2006).

\section{- Despesa total com saúde por habitante}

Representa o somatório do gasto em saúde por habitante no município com recursos próprios 
e com transferências do Sistema Único de Saúde (SUS). O dado refere-se ao ano 2002 e é proveniente do Sistema de Informações sobre Orçamentos Públicos em Saúde (SIOPS - http://siosp. datasus.gov.br, acessado em 20/Mar/2006).

\section{- Médicos por mil habitantes}

Refere-se à dimensão de recursos humanos disponíveis no município com potencialidade de atuar diretamente no desfecho investigado. $\mathrm{O}$ ano de referência é 200011.

As taxas de mortalidade infantil por causas evitáveis foram estratificadas em quartis, sendo o primeiro quartil aquele com menor coeficiente. Para testar a normalidade da distribuição das variáveis investigadas, utilizou-se o teste de Kolmogorov-Smirnov. Para aquelas variáveis sem aderência à normalidade, a associação entre as variáveis foi testada pelo coeficiente de correlação de Spearman, e utilizado o coeficiente de correlação de Pearson para as variáveis com distribuição normal. O teste ANOVA e seu correspondente não paramétrico, o teste de Kruskal-Wallis, foram empregados para identificar diferenças significativas entre os quartis de mortalidade infantil por causas evitáveis segundo as variáveis independentes. Em todos os testes, foi utilizado como ponto de corte para rejeição da hipótese nula um p valor $<0,05$. Todas as variáveis também foram analisadas segundo as regiões do país. Os municípios das regiões Sul e Sudeste, que apresentam os melhores níveis sócio-econômicos e de saúde 12 , compuseram um grupo, e os municípios das regiões Centro-Oeste, Norte e Nordeste foram reunidos em outro conjunto. Padrões distintos entre esses dois grupos de regiões vêm sendo descritos na literatura em relação a outros desfechos em saúde 13,14.

Para as análises estatísticas, foi utilizado o programa Stata 9 (Stata Corp., College Station, Estados Unidos).

\section{Resultados}

Entre os anos 2000 e 2002 ocorreram 63.265 óbitos por causas evitáveis em crianças menores de um ano nos municípios brasileiros com mais de 80 mil habitantes. Os valores foram declinantes, passando de 13,05 óbitos a cada mil nascidos vivos em 2000 para 11,28 em 2002. A Tabela 1 apresenta a estatística descritiva de todas as variáveis. Apenas o CMI, o coeficiente de Gini, o PIB per capita e o número de estabelecimentos de saúde por 10 mil habitantes apresentaram distribuição normal. Com relação ao desfecho, houve acentuada variação entre o valor máximo (Açailândia, Maranhão; 31,01/mil nascidos vivos) e o valor mínimo (Bento Gonçalves, Rio Grande do Sul; $4,01 /$ mil nascidos vivos), uma diferença de quase oito vezes. Também as variáveis independentes apresentaram marcantes desigualdades entre os valores extremos.

Os coeficientes de correlação apresentados na Tabela 2 indicam a existência de correlação negativa estatisticamente significante da mortalidade infantil por causas evitáveis com o IDHM, o PIB per capita, a proporção de pessoas que vivem domicílios com banheiro e água encanada, a despesa total com saúde por habitante e o número

Estatística descritiva dos indicadores sócio-econômicos e de saúde. Municípios brasileiros com mais de 80 mil habitantes, $2000-2002$ ( $\mathrm{N}=296$ ).

\begin{tabular}{|c|c|c|c|c|c|c|}
\hline Variáveis & Média (DP) & Mediana & Mínimo-máximo & Quartil 25 & Quartil 75 & $\mathrm{p}$ valor * \\
\hline $\mathrm{CMI}$ & $12,402(4,480)$ & 11,730 & $4,010-31,010$ & 9,118 & 14,838 & 0,068 \\
\hline IDHM & $0,780(0,054)$ & 0,790 & $0,558-0,919$ & 0,749 & 0,818 & 0,002 \\
\hline Gini & $0,562(0,053)$ & 0,560 & $0,430-0,690$ & 0,520 & 0,600 & 0,189 \\
\hline PIB per capita & $304,560(130,358)$ & 291,045 & $71,060-834,000$ & 210,025 & 382,585 & 0,104 \\
\hline Acesso a serviço básico & $85,615(17,562)$ & 93,240 & $12,320-99,190$ & 81,115 & 97,115 & $<0,001$ \\
\hline $\begin{array}{l}\text { Despesa total com saúde } \\
\text { por habitante }\end{array}$ & $126,353(64,506)$ & 118,430 & $8,710-426,970$ & 73,152 & 167,958 & 0,019 \\
\hline $\begin{array}{l}\text { Estabelecimentos de } \\
\text { saúde por } 10 \text { mil habitantes }\end{array}$ & $3,405(1,622)$ & 3,248 & $0,520-10,230$ & 2,050 & 4,387 & 0,460 \\
\hline Médicos por mil habitantes & $1,077(1,052)$ & 0,760 & $0,000-7,270$ & 0,320 & 1,520 & $<0,001$ \\
\hline
\end{tabular}

CMI: coeficiente de mortalidade infantil; IDHM: índice de desenvolvimento humano municipal; PIB: produto interno bruto.

* Teste de Kolmogorov-Smirnov. 
Matriz de correlação entre os indicadores sócio-econômicos e de saúde. Municípios brasileiros com mais de 80 mil habitantes, $2000-2002$.

\begin{tabular}{|c|c|c|c|c|c|c|c|c|}
\hline & CMI & IDHM & Gini & $\begin{array}{l}\text { PIB } \\
\text { per } \\
\text { capita }\end{array}$ & $\begin{array}{c}\text { Acesso a } \\
\text { serviço } \\
\text { básico }\end{array}$ & $\begin{array}{l}\text { Despesa total } \\
\text { com saúde } \\
\text { por habitante }\end{array}$ & $\begin{array}{l}\text { Estabelecimentos } \\
\text { de saúde por } \\
10 \text { mil habitantes }\end{array}$ & $\begin{array}{c}\text { Médicos } \\
\text { por mil } \\
\text { habitantes }\end{array}$ \\
\hline $\mathrm{CMI}$ & 1 & $-0,645$ * & 0,394 * & $-0,512$ * & $-0,590$ * & $-0,244$ * & $-0,058$ & $-0,247$ * \\
\hline IDHM & & 1 & $-0,216$ * & 0,865 * & 0,796 * & 0,446 * & 0,189 * & 0,618 * \\
\hline Gini & & & 1 & $-0,12$ & $-0,471$ * & 0,114 & 0,323 * & 0,343 * \\
\hline PIB per capita & & & & 1 & 0,767 * & 0,493 * & 0,250 * & 0,759 * \\
\hline Acesso a serviço básico & & & & & 1 & 0,383 * & 0,061 & 0,452 * \\
\hline \multicolumn{9}{|l|}{ Despesa total com saúde } \\
\hline por habitante & & & & & & 1 & 0,214 * & 0,493 * \\
\hline \multicolumn{9}{|l|}{ Estabelecimentos de saúde } \\
\hline por 10 mil habitantes & & & & & & & 1 & 0,520 * \\
\hline Médicos por mil habitantes & & & & & & & & 1 \\
\hline
\end{tabular}

CMI: coeficiente de mortalidade infantil; IDHM: índice de desenvolvimento humano municipal; PIB: produto interno bruto.

* Significativo no nível de $1 \%$.

de médicos por mil habitantes. Foi, ainda, identificada correlação positiva entre a mortalidade infantil por causas evitáveis e o coeficiente de Gini. Percebe-se também alta correlação positiva entre PIB per capita do município e o número de médicos por mil habitantes e PIB per capita e IDHM.

A distribuição das variáveis independentes segundo os quartis de mortalidade pode ser observada na Tabela 3. Os municípios que compuseram os quartis com maior mortalidade infantil por causas evitáveis apresentaram também menor IDHM ( $\mathrm{p}<0,001)$. O mesmo ocorre com o PIB per capita, com a proporção de pessoas que vivem em domicílios com banheiro e água encanada, com a despesa total com saúde por habitante e com o número de médicos por mil habitantes $(p<0,001)$. O inverso é observado com o coeficiente de Gini $(p<0,001)$.

A Figura 1 mostra a distribuição conjunta da mortalidade infantil por causas evitáveis segundo os quartis de PIB per capita e de distribuição de renda (coeficiente de Gini). Quando se compara o grupo de municípios que está no quartil de melhor distribuição de renda (quartil 1) e também de maior PIB per capita (quartil 4) com o grupo antagônico, ou seja, com a pior distribuição de renda e menor PIB per capita vê-se que há uma acentuada sobre mortalidade no município. Enquanto no primeiro a média de mortalidade infantil por causas evitáveis é igual a 7,3 por mil nascidos vivos, no último o coeficiente equivale a 18,2 , isto é, uma sobremortalidade de aproximadamente $150 \%$.

A desigualdade geográfica na distribuição dos indicadores investigados também é evidente
(Tabela 4). Os municípios das regiões Norte, Nordeste e Centro-Oeste agrupados tiveram maior mortalidade infantil por causas evitáveis e piores indicadores sócio-econômicos e de investimento e serviços de saúde em relação ao grupo que reuniu os municípios das regiões Sul e Sudeste. Quanto à mortalidade, o coeficiente é aproximadamente $40 \%$ superior no primeiro grupo quando comparado ao segundo.

\section{Discussão}

A análise de informações geradas mediante dados secundários sempre requer cautela. Em particular a construção do coeficiente de mortalidade infantil, que é bastante sensível às limitações dos Sistemas de Informações em Saúde (SIS) brasileiros. O SIM, cujos dados foram utilizados no numerador do desfecho, apresenta como deficiências o preenchimento inadequado das Declarações de Óbitos e a subnotificação de mortes. No entanto, conta com grande cobertura, decorrente, sobretudo, pela notificação do óbito ser compulsória e pelo formulário de sua declaração ser distribuído gratuitamente 15 . Nos últimos anos o registro de óbitos no grupo de causas mal definidas tem sido reduzido em todo o Brasil, entretanto uma pior qualidade nos dados ainda é identificada nas regiões rurais do Norte e do Nordeste 12. O SINASC apresentou nos últimos anos uma acentuada melhoria na qualidade da informação gerada, além da destacada ampliação geográfica de sua cobertura 16 . Há, porém, a exemplo do SIM, problemas de subnotificação. 
Média dos indicadores sócio-econômicos e de saúde segundo os quartis do coeficiente de mortalidade infantil por causas evitáveis. Municípios brasileiros com mais de 80 mil habitantes, 2000-2002

\begin{tabular}{|c|c|c|c|c|c|}
\hline & $\begin{array}{c}\text { 1ㅇ quartil } \\
(4,010-9,118)\end{array}$ & $\begin{array}{c}\text { 2o quartil } \\
(9,119-11,730)\end{array}$ & $\begin{array}{c}\text { 3o quartil } \\
(11,731-14,838)\end{array}$ & $\begin{array}{c}\text { 4o quartil } \\
(14,839-31,010)\end{array}$ & $\mathrm{p}$ valor * \\
\hline IDHM & 0,820 & 0,797 & 0,771 & 0,733 & $<0,001$ \\
\hline Gini & 0,544 & 0,549 & 0,559 & 0,594 & $<0,001$ \\
\hline PIB per capita & 394,879 & 344,363 & 265,851 & 212,087 & $<0,001$ \\
\hline Acesso a serviço básico & 94,273 & 90,785 & 86,414 & 70,929 & $<0,001$ \\
\hline Despesa total com saúde por habitante & 151,826 & 133,404 & 115,198 & 105,428 & $<0,001$ \\
\hline Estabelecimentos de saúde por 10 mil habitantes & 3,814 & 3,133 & 3,371 & 3,304 & 0,265 \\
\hline Médicos por mil habitantes & 1,425 & 1,289 & 0,842 & 0,746 & $<0,001$ \\
\hline
\end{tabular}

IDHM: índice de desenvolvimento humano municipal; PIB: produto interno bruto.

* Teste ANOVA; teste de Kruskal-Wallis.

A opção por incluir nesse estudo apenas os municípios brasileiros com mais de 80 mil habitantes procurou minimizar a interferência dessas limitações dos SIS nos resultados finais e também minorar a instabilidade das taxas que ocorre nos municípios de pequeno porte. De modo geral, são exatamente os municípios de maior porte os que apresentam melhor gestão das informações em saúde e assistência médica, acabando por produzir informações mais estáveis e fidedignas. Trabalhar com as informações oficiais dos sistemas - apesar de suas conhecidas limitações - também busca colaborar na discussão sobre a gestão dos dados e no processo de sua melhoria.

O decréscimo da mortalidade infantil ocorrido no Brasil, inclusive durante períodos de recessão econômica - como na década de 1990 - e o fato de em algumas regiões ter sido descrita uma similaridade na mortalidade infantil entre áreas com melhores e piores condições sociais, poderia revelar uma tendência de homogeneização das taxas de óbitos em menores de um ano entre os estratos sociais 17. O presente estudo, contudo, indica no nível ecológico a permanência de importantes desigualdades na distribuição dos óbitos infantis por causas evitáveis, configurando-os como bastante sensíveis às condições sócio-econômicas, aos investimentos públicos em saúde e à oferta de médicos nos municípios. Aqueles com um baixo índice de desenvolvimento humano, assim como com uma alta concentração de renda, baixa condição sanitária, reduzido PIB per capita, com menor despesa total com saúde por habitante e menor proporção de médicos por mil habitantes apresentaram maior taxa de mortalidade infantil por causas evitáveis em relação àqueles com melhores condições. De maneira
Figura 1

Coeficiente de mortalidade infantil (CMI) por causas evitáveis segundo os quartis de renda per capita e do coeficiente de Gini. Municípios brasileiros com mais de 80 mil habitantes, 2000-2002.

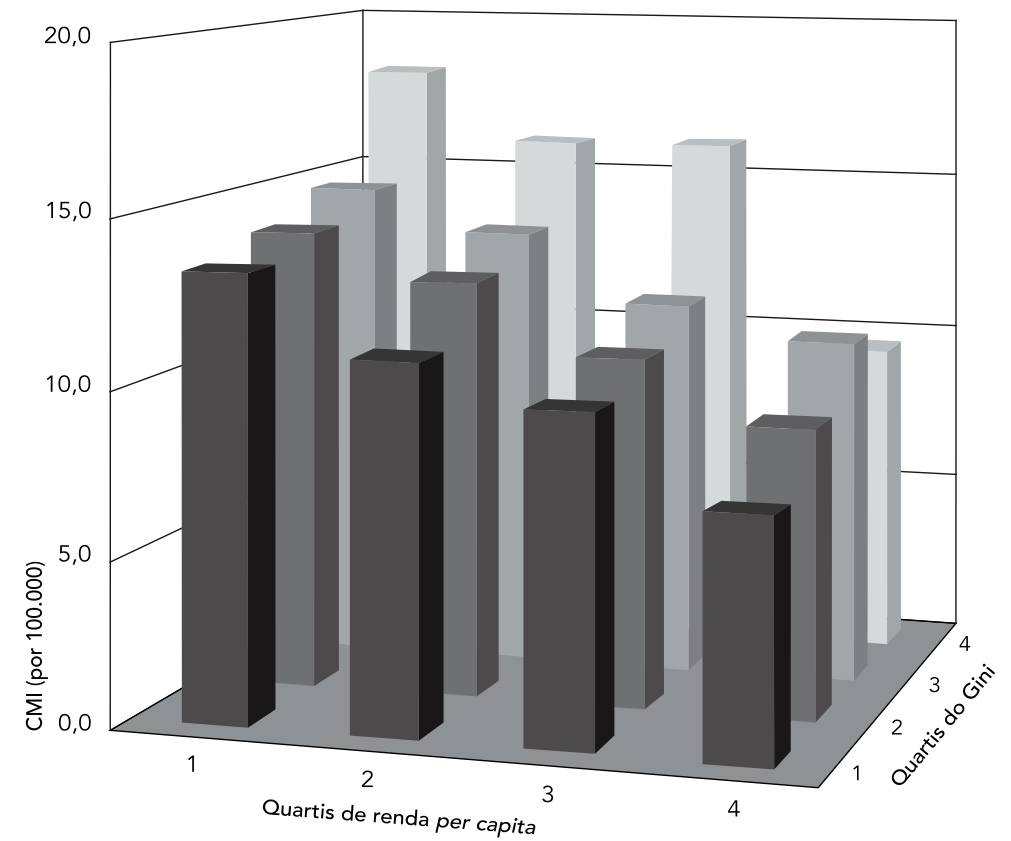


Média dos coeficientes de mortalidade infantil por causas evitáveis segundo as regiões do país. Municípios brasileiros com mais de 80 mil habitantes, 2000-2002

\begin{tabular}{|c|c|c|c|c|c|c|c|c|c|}
\hline & $\mathrm{n}$ & $\mathrm{CMI}$ & IDHM & Gini & $\begin{array}{c}\text { PIB } \\
\text { per capita }\end{array}$ & $\begin{array}{l}\text { Acesso a } \\
\text { serviço } \\
\text { básico }\end{array}$ & $\begin{array}{l}\text { Despesa } \\
\text { total com saúde } \\
\text { por habitante }\end{array}$ & $\begin{array}{l}\text { Estabelecimentos } \\
\text { de saúde por } \\
10 \text { mil habitantes }\end{array}$ & $\begin{array}{l}\text { Médicos } \\
\text { por mil } \\
\text { habitantes }\end{array}$ \\
\hline \multicolumn{10}{|l|}{ Norte + Nordeste } \\
\hline + Centro-Oeste & 99 & 15,374 & 0,731 & 0,601 & 208,539 & 67,789 & 105,292 & 3,321 & 0,708 \\
\hline Sul + Sudeste & 197 & 10,908 & 0,805 & 0,542 & 352,814 & 94,573 & 136,724 & 3,447 & 1,262 \\
\hline
\end{tabular}

CMI: coeficiente de mortalidade infantil; IDHM: índice de desenvolvimento humano municipal; PIB: produto interno bruto.

semelhante, agrupando os 399 municípios do Paraná em cinco clusters de acordo com o nível de condições de vida, Andrade et al. 18 descreveram que os municípios com melhores condições apresentaram menores taxas de mortalidade pós-neonatal e de mortalidade infantil. A redução da mortalidade infantil, portanto, ao menos quanto às decorrentes por causas evitáveis, não refletiu necessariamente na supressão das desigualdades. Possivelmente foram os municípios mais ricos e os estratos sociais mais privilegiados que se beneficiaram inicialmente das estratégias de redução dos óbitos infantis.

Desigualdades espaciais na mortalidade infantil também foram relatadas no ambiente intra-urbano. Guimarães et al. 19 construíram por intermédio dos dados oriundos do censo de 1991 um indicador sintético das condições de vida dos bairros de Recife e encontraram menor mortalidade infantil, neonatal e pós-neonatal nos estratos de melhor condição de vida. Costa et al. 20 investigaram a distribuição espacial da mortalidade infantil proporcional no Município de Salvador e encontraram correlação positiva entre este indicador e baixa renda, proporção de casas em favelas e quantidade de centros de saúde; correlação negativa foi descrita entre mortalidade infantil proporcional e nível educacional e proporção de casas ligadas à rede de abastecimento de água.

Quando as unidades de análise são os indivíduos, as desigualdades na ocorrência dos óbitos infantis também são encontradas e influenciadas por variáveis sócio-econômicas. Andrade et al. 21, ao analisarem o percentual de óbitos perinatais no Rio de Janeiro segundo variáveis sócio-econômicas coletadas junto às parturientes, identificaram maiores taxas de mortalidade entre as mães com menor grau de escolaridade e entre os grupos cujo chefe da família apresentava menor renda. Causas evitáveis de mortalidade infantil também se mostraram associadas com existência de eletrodomésticos no domicílio e o nível de escolaridade materna e paterna 22 . Piores condições sócio-econômicas, de maneira mais proximal, podem estar acarretando deficiências nutricionais, maior exposição a traumas e a agentes infecciosos, além de menor acesso a informações e serviços de saúde.

No presente estudo foi ainda identificada uma distribuição geográfica desigual entre as macrorregiões do Brasil para todas as variáveis - independentes e desfecho -, e os municípios das regiões Norte, Nordeste e Centro-Oeste concentraram os piores indicadores. Esse fenômeno não é novidade no Brasil. Szwarcwald et al. ${ }^{8}$, ao analisarem a evolução da mortalidade infantil no país na década de 1980 dividiram o território nacional em três grupos e identificaram no mais pobre um padrão de mortalidade infantil similar à Índia; o grupo oposto apresentava melhores indicadores, todavia ainda distantes dos observados nos países desenvolvidos. Os autores também apontaram os estados das regiões Nordeste e Norte como aqueles com piores situações de mortalidade infantil. A análise comparativa das regiões do Brasil indicou a existência, ainda no início do século XXI, de marcantes desigualdades espaciais, apesar de já serem descritas na literatura há décadas. Políticas públicas que promovam o desenvolvimento equânime do país precisam ser implementadas, minimizando-se as diferenças entre as regiões.

O presente estudo identificou associação negativa da mortalidade infantil por causas evitáveis com o investimento per capita em saúde e o número de médicos por mil habitantes. É difícil, no entanto, dissociar a distribuição desta variável da situação sócio-econômica dos municípios, uma vez que há concentração de médicos nas regiões com melhores condições de vida, sobretudo onde há maior PIB per capi- 
ta. Já o número de estabelecimentos de saúde por 10 mil habitantes no município não apresentou diferença estatisticamente significante entre os quartis do desfecho. Esse resultado pode ser explicado pelo fato de que um maior número desses estabelecimentos no município não necessariamente reflete uma melhor atenção primária ou um setor de média e alta complexidade melhor estruturado e equipado. A importância da atenção primária em saúde na mortalidade infantil também é descrita por Macinko et al. 23 que, ao avaliarem o impacto do Programa Saúde da Família nesse desfecho, entre 1990 e 2002, descreveram que um aumento de $10 \%$ na cobertura do programa foi associado com uma queda de $4,5 \%$ no coeficiente de mortalidade infantil do país. Além da qualidade da assistência médica, a descentralização do sistema de saúde tem sido descrita como importante fator na redução dos óbitos infantis evitáveis 23 . Campos et al. 24 descrevem, inclusive, que no Município do Rio de Janeiro houve redução do impacto das condições de vida na mortalidade infantil ao longo das últimas décadas em função do aumento de importância de programas de vacinação, reidratação oral e atendimento às infecções respiratórias agudas. Porém, ao analisarem dados de duas coortes de nascidos vivos em
Pelotas, Rio Grande do Sul, Costa et al. 25 afirmaram que os serviços de saúde não beneficiam aqueles que mais necessitam de sua atenção, mas sim que concentram esforços nas populações que deles menos dependem. Assim, o seu potencial papel de reduzir mortes e desigualdades fica parcialmente prejudicado. Preocupante também é que em diversos países o aumento no gasto global em saúde tem ocorrido principalmente por um acréscimo no gasto privado, o que poderá causar uma distorção ainda maior no tocante à eqüidade do sistema de saúde.

Reduzir a taxa de mortalidade infantil por causas evitáveis no país é fundamental, entretanto há desafios adicionais. Um deles é diminuir a desigualdade na distribuição dos óbitos entre os estratos sociais e as regiões geográficas. Olhares e ações iguais para realidades distintas apenas acentuarão as desigualdades, havendo necessidade, portanto, de políticas públicas coerentes com cada realidade. A alocação de recursos e o desenvolvimento de estratégias sociais e de saúde devem levar essa realidade em consideração. A compreensão do papel protagonista das condições de vida sobre a mortalidade infantil por causas evitáveis também deve permear as ações que visem à minimização da magnitude e da desigualdade dos óbitos infantis evitáveis. 


\section{Resumo}

O objetivo desse estudo foi testar as associações entre a mortalidade infantil por causas evitáveis no Brasil com indicadores sócio-econômicos e de serviços e investimentos em saúde. Trata-se de um estudo ecológico, cujas unidades de análise foram os 296 municípios brasileiros com população superior a 80 mil habitantes. Realizaram-se os testes de Kruskall-Wallis e ANOVA para identificar diferenças significativas entre os quartis de mortalidade infantil segundo as variáveis independentes, e foram calculados os coeficientes de correlação de Pearson e de Spearman para testar as associações entre todas as variáveis. Os municípios que compuseram os quartis com maior mortalidade infantil por causas evitáveis apresentaram também menor Índice de Desenvolvimento Humano Municipal, Produto Interno Bruto per capita, pessoas que vivem em domicílios com banheiro e água encanada, despesa total com saúde por habitante e médicos por mil habitantes; e maior coeficiente de Gini. A compreensão do papel protagonista das condições sócio-econômicas e de investimentos em saúde sobre a mortalidade infantil por causas evitáveis deve permear as ações que visem à minimização da magnitude e da desigualdade desses óbitos.

Mortalidade Infantil; Coeficiente de Mortalidade Infantil; Serviço de Saúde; Fatores Sócio-Econômicos

\section{Colaboradores}

A. F. Boing trabalhou na concepção da pesquisa, na coleta e análise dos dados, na pesquisa bibliográfica e na redação do artigo. A. C. Boing atuou na concepção da pesquisa, na pesquisa bibliográfica e na redação e revisão crítica do artigo.

\section{Referências}

1. Rosano A, Botto LD, Botting B, Mastroiacovo P. Infant mortality and congenital anomalies from 1950 to 1994: an international perspective. J Epidemiol Community Health 2000; 54:660-6.

2. Fundo das Nações Unidas para a Infância. Situação da infância brasileira 2006. Brasília: Fundo das Nações Unidas para a Infância; 2006.

3. Monteiro CA, Nazário CL. Declínio da mortalidade infantil e equidade social: o caso da cidade de São Paulo entre 1973 e 1993. In: Monteiro CA, organizador. Velhos e novos males da saúde no Brasil. São Paulo: Editora Hucitec; 1995. p. 173-85.

4. Maranhão AGK, Joaquim MMC, Siu C. Mortalidade perinatal e neonatal no Brasil. Tema 1999; 17:6-17.

5. Costa MCN, Mota ELA, Paim JS, Silva LMV, Teixeira MV, Mendes CMC. Mortalidade infantil no Brasil em períodos recentes de crise econômica. Rev Saúde Pública 2003; 37:699-706.

6. Diamond I. Child mortality - the challenge now. Bull World Health Organ 2000; 78:1174.
7. Wagstaff A. Socioeconomic inequalities in child mortality: comparisons across nine developing countries. Bull World Health Org 2000; 78:19-29.

8. Szwarcwald CL, Leal MC, Castilho EA, Andrade CLT. Mortalidade infantil no Brasil: Belíndia ou Bulgária? Cad Saúde Pública 1997; 13:503-16.

9. Pochmann M, Amorim R. Atlas da exclusão social no Brasil. São Paulo: Cortez Editora; 2003.

10. Brasil. Portaria $n^{\circ} .1 .121$. Estabelece os mecanismos, fluxos e prazos para a avaliação de desempenho em relação às metas municipais e estaduais definidas no Pacto de Indicadores da Atenção Básica. Diário Oficial da União 2002; 17 jun.

11. Programa das Nações Unidas para o Desenvolvimento. Atlas do desenvolvimento humano no Brasil. Brasília: Programa das Nações Unidas para o Desenvolvimento; 2003.

12. Mello-Jorge MHP, Gotlieb SLD. As condições de saúde no Brasil. Rio de Janeiro: Editora Fiocruz; 2000 . 
13. Carmo EH, Barreto ML, Silva Jr. JB. Mudanças nos padrões de morbimortalidade da população brasileira: os desafios para um novo século. Epidemiol Serv Saúde 2003; 12:63-75.

14. Wünsch-Filho V. The epidemiology of oral and pharynx cancer in Brazil. Oral Oncol 2002; 35:73746.

15. Carvalho D M. Grandes sistemas nacionais de informação em saúde: revisão e discussão da situação atual. Inf Epidemiol SUS 1997; 4:8-46.

16. Ministério da Saúde. Saúde Brasil 2004: uma análise da situação de saúde. Brasília: Ministério da Saúde; 2004.

17. Goldani MZ, Benatti R, Silva AAM, Bettiol H, Correa JCW, Tietzmann M. Narrowing inequalities in infant mortality in Southern Brazil. Rev Saúde Pública 2002; 36:478-83.

18. Andrade SM, Soares DA, Matsuo T, Souza RKT, Mathias TAF, Iwakura MLH, et al. Condições de vida e mortalidade infantil no Estado do Paraná, Brasil, 1997/2001. Cad Saúde Pública 2006; 22:181-9.

19. Guimarães MJB, Marques NM, Melo Filho DJ, Szwarcwald CL. Condições de vida e mortalidade infantil: diferenciais intra-urbanos no Recife, Pernambuco, Brasil. Cad Saúde Pública 2003; 19:141324.

20. Costa MCN, Azi PA, Paim JS, Silva LMV. Mortalidade infantil e condições de vida: a reprodução das desigualdades sociais em saúde na década de 90 . Cad Saúde Pública 2001; 17:555-67.
21. Andrade CLT, Szwarcwald CL, Gama SGN, Leal MC Desigualdades sócio-econômicas do baixo peso ao nascer e da mortalidade perinatal no Município do Rio de Janeiro, 2001. Cad Saúde Pública 2004; 20 Suppl 1:S44-51.

22. França E, Souza JM, Guimarães MDC, Goulart EMA, Colosimo E, Antunes CMF. Associação entre fatores sócio-econômicos e mortalidade infantil por diarréia, pneumonia e desnutrição em região metropolitana do Sudeste do Brasil: um estudo caso-controle. Cad Saúde Pública 2001; 17:1437-47.

23. Macinko J, Guanais FC, Souza MFM. Evaluation of the impact of the Family Health Program on infant mortality in Brazil, 1990-2002. J Epidemiol Community Health 2006; 60:13-9.

24. Campos TP, Carvalho MS, Barcellos CC. Mortalidade infantil no Rio de Janeiro, Brasil: áreas de risco e trajetória dos pacientes até os serviços de saúde. Rev Panam Salud Publica 2000; 8:164-71.

25. Costa JSD, Victora CG, Barros FC, Halpern R, Horta BL, Manzolli P. Assistência médica materno-infantil em duas coortes de base populacional no Sul do Brasil: tendências e diferenciais. Cad Saúde Pública 1996; 12 Suppl 1:S59-66.

Recebido em 14/Jul/2006

Versão final reapresentada em 09/Abr/2007

Aprovado em 13/Jul/2007 